University of New Jersey, Piscataway, NJ, USA. ${ }^{2}$ Protein Data Bank in Europe (PDBe), European Molecular Biology Laboratory, European Bioinformatics Institute (EMBL-EBI), Wellcome Genome Campus, Hinxton, UK. ${ }^{3}$ Protein Data Bank Japan (PDBj), Institute for Protein Research, Osaka University, Osaka, Japan.

凶e-mail: jasmine.young@rcsb.org

Published online: 7 May 2021

https://doi.org/10.1038/s41592-021-01137-z
References

1. Howe, D. et al. Nature 455, 47-50 (2008).

2. Young, J. Y. et al. Database 2018, bay002 (2018).

3. Wilkinson, M. D. et al. Sci. Data 3, 160018 (2016).

4. Berman, H., Henrick, K. \& Nakamura, H. Nat. Struct. Biol. 10, 980 (2003).

5. wwPDB Consortium. Nucleic Acids Res. 47, D520-D528 (2019).

\section{Acknowledgements}

RCSB PDB is jointly funded by the US National Science

Foundation (DBI-1832184), the US Department of Energy (DE-SC0019749) and the National Cancer Institute,

National Institute of Allergy and Infectious Diseases and

National Institute of General Medical Sciences of the
US National Institutes of Health (R01GM133198). The Protein Data Bank in Europe is supported by the European Molecular Biology Laboratory-European Bioinformatics Institute and Wellcome Trust (104948). Protein Data Bank Japan is supported by the Database Integration Coordination Program from the National Bioscience Database Center (NBDC)-JST (Japan Science and Technology Agency), the Platform Project for Supporting in Drug Discovery and Life Science Research from AMED, and the joint usage program of Institute for Protein Research, Osaka University.

Competing interests

The authors declare no competing interests.

\title{
A new era of synchrotron-enabled macromolecular crystallography
}

The future of macromolecular crystallography includes new X-ray sources, enhanced remote-accessible capabilities and time-resolved methods to capture intermediate structures along reaction pathways.

\section{Aina E. Cohen}

n 1974, three years after the official start of the Protein Data Bank, the first beamlines dedicated to structural studies began operation at multi-GeV storage rings. During early macromolecular crystallography experiments, a crystal exposed for only a few minutes would produce a higher resolution diffraction pattern than could be measured after hours with a rotating anode source, first demonstrating the value of synchrotron radiation to structural biology ${ }^{1}$. At state-of-the-art synchrotron beamlines today, complete datasets are measured in seconds with crystal rotation speeds exceeding $90^{\circ} \mathrm{s}^{-1}$ and diffraction image frame rates exceeding $100 \mathrm{~Hz}$. The resulting images are monitored for diffraction quality in real time and transferred to automated processing pipelines to simplify data analysis. As technologies advance, structural investigations are transitioning beyond solving single static structures. Sequential series of structural snapshots are being applied to provide details of the atomic positions and motions that define the relationships involved in molecular recognition, transition state stabilization, allostery and other aspects of the biocatalytic process.

Many proteins retain biological function in the crystalline state, and macromolecular crystallography offers opportunities to study these in different environments and temperatures. Brighter microbeam sources have enabled the use of smaller samples

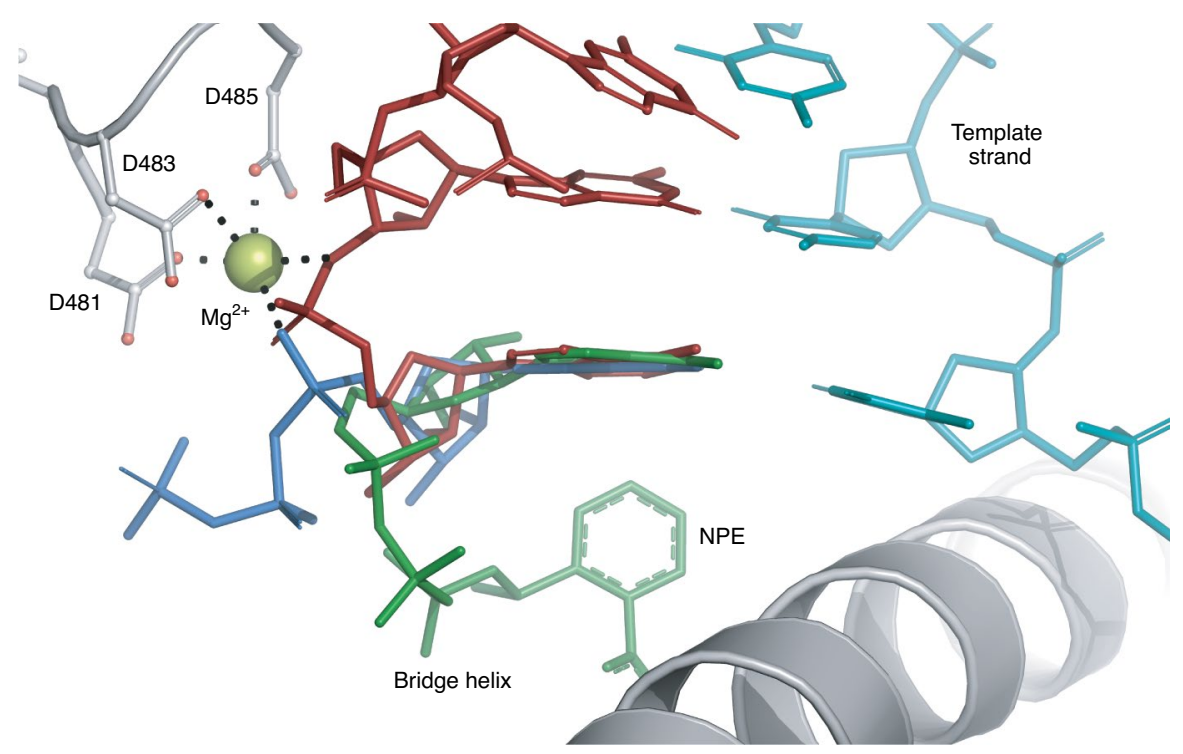

Fig. 1 | Time-resolved studies of transcription. The Calero lab (University of Pittsburgh) has demonstrated that photoactive caged ATP (green) can bind to RNA polymerase II and that ultraviolet illumination can break the nitrobenzyl group (NPE) in crystallo, allowing ATP release, metal coordination (blue) and phosphodiester bond formation (red). These experiments were performed remotely using SSRL beam line 12-1, where crystals were exposed to UV light to break the cage, followed by a temperature increase from $100 \mathrm{~K}$ to $170 \mathrm{~K}$ (above the glass transition temperature of water), followed by rapid helical-rotation data collection (2 s per dataset). Credit:Guillermo Calero.

that have improved diffusion and optical transmission characteristics, supporting burgeoning methods for rapid microcrystal freeze-trapping, whereby a process of interest is initiated using light or chemical mixing to capture intermediate structures along the reaction pathway. However, it is extremely difficult to freeze-capture events faster than $\sim 10 \mathrm{~ms}$. To observe faster reactions, time-resolved methods 
that initiate and monitor reactions 'on the fly' are required. Here emergent macromolecular crystallography methods and next-generation X-ray sources are having an increasing impact-in particular, to study structures at physiologically relevant temperatures.

While sample cryopreservation was an important technological leap for synchrotron radiation macromolecular crystallography, multicrystal strategies are a powerful alternative approach to overcoming the limitations of radiation damage. At an X-ray free electron laser, a short (5-50 fs) X-ray pulse will produce a still diffraction pattern before substantial radiation-induced atomic rearrangements occur within the crystal. This technology has helped expand the structural information accessible from small radiation-sensitive crystals at room temperature and enable ultra-fast time-resolved measurements. However, because the sample is destroyed during this process, a series of crystals must be exposed to obtain a complete dataset, in a process termed serial crystallography ${ }^{2}$. By spreading the X-ray dose over many crystals, the application of serial crystallography methods ${ }^{3}$ at the synchrotron enables structural studies with smaller crystals and time-resolved measurements of irreversible reactions. When crystals can survive multiple exposures, rapid serial rotation methods are possible, in which multiple time points are probed from each crystal using a detector with a fast frame rate. The resulting data are analyzed with powerful new algorithms originally developed for X-ray free electron laser experiments. Time-resolved macromolecular crystallography at the synchrotron can provide atomic resolution structures at microsecond temporal resolution using monochromatic X-rays, or sub-nanosecond resolution using pink beam single-bunch operational modes; new sources that incorporate pseudo-single-bunch orbits open up possibilities for routine measurements in this regime ${ }^{4}$. Time-resolved macromolecular crystallography studies at room temperature enable visualization of functionally relevant water networks and alternative conformations disrupted by cryopreservation. The conformational flexibility retained at room temperature provides greater access to ligand binding pockets, facilitating binding studies and fragment-based drug discovery ${ }^{5}$. Intermediate state structures from time-resolved measurements can provide electronic and spatial features to aid in the modeling of new drug-like compounds.

The power of macromolecular crystallography at synchrotrons to facilitate the development of new therapeutics and to reveal the interactions involved for their mechanisms is exemplified by the rapid response of structural biologists to the COVID-19 pandemic, who collectively have made over 800 SARS-CoV-2-related deposits to the Protein Data Bank, with dozens of associated drugs in clinical trials. To enable COVID-19related science at room temperature during a period of restricted access, new in situ crystallization and remote data collection schemes have been released at the Stanford Synchrotron Radiation Lightsource that avoid direct manipulation of crystals, support robotic sample exchange and allow full rotational access of the sample in a controlled-humidity environment. By simplifying room temperature crystal handling and transport, technologies of this nature are an important first step toward bringing time-resolved macromolecular crystallography into general use. During next-generation experiments, remote researchers may control the timing of photo-triggered serial rotation measurements (Fig. 1) and may access drop-on-demand systems to initiate serial crystal mixing. The pace of these innovations will continue to accelerate as synchrotron science begins a new era of discovery with an increased emphasis on uncovering the relationships of molecular motion to biological function.

\section{Aina E. Cohen $\times$}

Stanford Synchrotron Radiation Lightsource, SLAC National Accelerator Laboratory, Stanford University, Menlo Park, CA, USA.

$凶_{e-\text { mail:ACohen@slac.stanford.edu }}$

Published online: 7 May 2021

https://doi.org/10.1038/s41592-021-01146-y

References

1. Phillips, J. C., Wlodawer, A., Yevitz, M. M. \& Hodgson, K. O. Proc. Natl Acad. Sci. USA 73, 128-132 (1976).

2. Chapman, H. N. et al. Nature 470, 73-77 (2011).

3. Martiel, I., Müller-Werkmeister, H. M. \& Cohen, A. E. Acta Crystallogr. D Struct. Biol. 75, 160-177 (2019).

4. Sun, C., Robin, D. S., Steier, C. \& Portmann, G. Phys. Rev. Accel. Beams 18, 120702 (2015).

5. Fischer, M., Shoichet, B. K. \& Fraser, J. S. ChemBioChem 16, 1560-1564 (2015)

\section{From structure to mechanism: skiing the energy landscape}

It is time for structural biologists to embrace the challenge of quantitatively describing functional energy landscapes.

\section{Dorothee Kern}

M odern structural biology, driven by X-ray crystallography, electron microscopy, nuclear magnetic resonance (NMR) and computational structure prediction, has revolutionized the visualization of biological macromolecules at atomic resolution. The speed of structure determination, the accuracy, the increasing complexity and size, and finally the beauty of biological structures are simply mind-blowing Yet such static snapshots usually do not adequately address the question of function, as they primarily capture the most populated, lowest energy structures. 